\title{
LAPAROTOMÍA DE GIORDANO-C HERNEY EN LA OPERACIÓN CESÁREA. UNA ALTERNATIVA EN LAS MALFORMACIONES DEL SISTEMA NERVIOSO CENTRAL FETAL
}

\author{
Harald Riesle P. ${ }^{1}$, Erasmo Bravo O. ${ }^{1}$, Aníbal Scarella C. ${ }^{1}$, Ignacio De la Torre $C .{ }^{1}$, Rolando \\ Márquez A. ${ }^{1}$, Bernardita Alamos L. ${ }^{a}$ \\ ${ }^{1}$ Departamento de Ginecología y Obstetricia, Universidad de Valparaíso. Servicio de Ginecología y Obstetricia, Hospital \\ Carlos Van Buren.
}

a Interna de Medicina, Universidad de Valparaíso.

\section{RESUMEN}

Las malformaciones del sistema nervioso central son la segunda malformación más frecuente en recién nacidos. La elección de la vía de parto es controversial. Nuestro centro ha adoptado el parto cesárea como de elección para resolver tales casos mediante laparotomía tipo Pfannenstiel y longitudinal media infraumbilical. La observación de algunas dificultades que ambas técnicas presentan nos llevó a adaptar, un abordaje extraído de la oncoginecología, a la práctica obstétrica. Presentamos 7 casos de malformaciones del sistema nervioso central resueltos satisfactoriamente mediante laparotomía de GiordanoCherney. La cirugía se realizó en forma electiva con un tiempo promedio de 72 minutos. No se registraron complicaciones en el intra o post operatorio. El manejo del dolor se realizó en forma rutinaria. Todas las pacientes evolucionaron favorablemente. No se presentaron complicaciones en los recien nacidos atribuibles a la cirugía.

\section{PALABRAS CLAVES: Malformaciones del sistema nervioso central, laparotomía de Giordano-Cherney}

\section{SUMMARY}

The central nervous system malformations are the second more frequent malformation in new born. The election of the way of delivery is controversial. Our center has adopted the cesarean section to resolve such cases, using either a Pfannenstiel laparotomy or a longitudinal infraumbilical section. The observation of some difficulties in both techniques carried us to adapt, a oncoginecological technique, to the obstetric practice. We present 7 cases with central nervous malformations resolved satisfactorily using a GiordanoCherney laparotomy. The surgery was carried out in elective form, with an average time of 72 minutes. No complications were registered intra or post surgery. The pain management was carried out in routine form. All patients evolved satisfactorily. No complications in new born where attributed to surgery.

KEY WORDS: Central nervous system malformation, Giordano-Cherney laparatomy 


\section{INTRODUCCIÓN}

Aproximadamente 2 a 3 de cada 100 recién nacidos (RN) son portadores de alguna anomalía congénita (1). En Chile, las malformaciones del sistema nervioso central son la segunda en frecuencia, con una prevalencia de 2,6 por cada 1.000 nacidos vivos (2). Entre las malformaciones más frecuentes se encuentran la hidrocefalia, el mielomeningocele, la microcefalia, el encefalocele, etc.

La vía de parto tras el diagnóstico antenatal de malformación del sistema nervioso central (SNC) es controversial. Actualmente se considera el parto vía cesárea, superior al vaginal, al disminuir el riesgo de daño fetal durante la extracción $(3,4,5,6,7)$.

Nuestro centro ha adoptado tal conducta y todo feto con diagnóstico antenatal de malformación del SNC se resuelve por vía alta utilizando una incisión transversa tipo Pfannenstiel, o una longitudinal media infraumbilical. Hemos observado que ambas presentan objeciones. Los criterios de una incisión abdominal satisfactoria considera que: permita una adecuada exposición, sea simple, extensible, conserve las estructuras de la región para facilitar la cicatrización, produzca un mínimo disconfort y lleve a una cicatriz cosméticamente aceptable.

Hasta 1900 se utilizaron exclusivamente las incisiones verticales (8). Estas permiten una rápida y adecuada exposición del campo (9), pero se asocian a un mayor riesgo dehiscencia de herida operatoria (9) y a una mala cosmética (8). Las incisiones transversales se desarrollaron para prevenir la dehiscencia de la fascia y la herniación de la pared. Actualmente más del $90 \%$ de las laparotomías en obstetricia son transversales (8). Pfannenstiel (10) popularizó este abordaje ya que satisface cabalmente los últimos 5 requisitos señalados. Sin embargo, su exposición del campo es limitada, por lo que la laparotomía longitudinal conserva ciertas indicaciones. Tal es el caso de las malformaciones del SNC, en que la necesidad de una adecuada exposición del campo quirúrgico, muchas veces contraindica la incisión tipo Pfannenstiel.

La incisión de Cherney (11) fue descrita y publicada por Leonid Cherney el año 1941 para las grandes intervenciones de abdomen inferior. Sin embargo, David Giordano en 1896 describe la misma incisión al realizar una laparotomía por cáncer. Por ello, conservamos ambos nombres denominándola Giordano-Cherney. Ésta se ha utilizado satisfactoriamente en cirugías ginecológicas, al recoger lo mejor de las incisiones trasversales pero corrigiendo su limitada exposición del campo quirúrgico. Su aplicación en la obstetricia es limitada, Crovetto (12) en 1957 la presentó como una opción en la operación cesárea de rutina. Desde entonces ha sido olvidada en la práctica obstétrica.

\section{PACIENTES Y MÉTODOS}

Presentamos un estudio retrospectivo de pacientes sometidas a operación cesárea, según técnica de Giordano-Cherney, que contaban con diagnóstico antenatal de malformación del SNC. Se analizaron 7 casos. Registramos los datos obtenidos de las fichas clínicas, considerando la edad de la paciente, diagnóstico antenatal, morbilidad, tiempo operatorio, necesidad de transfusión, manejo del dolor y complicaciones. La indicación quirúrgica fue el diagnóstico antenatal de hidrocefalia en 4 casos, encefalocele en 2 casos y en la paciente restante mielomeningocele.

Técnica quirúrgica. La incisión curvilínea y cóncava se inicia $1 \mathrm{~cm}$ por dentro y por debajo de la espina iliaca antero superior, cruza la línea media sobre la línea del vello púbico y termina en el lado opuesto simétricamente. La incisión del tejido subcutáneo continúa hasta la exposición de la vaina de los rectos. La vaina anterior de los rectos es abierta a cada lado de la línea alba, 3 centímetros sobre el pubis. Se abre la vaina lateralmente y continuamos separando en el mismo sentido el tendón conjunto del oblicuo mayor y menor cuyas fibras corren paralelas a la sección, en un principio unidas para luego separarse en dos hojas distintas. En algunos casos se expone también las fibras musculares de los oblicuos. En este tiempo quirúrgico deben ligarse y seccionarse las arterias epigástricas inferiores, que se ubican por debajo y hacia los bordes laterales de los rectos. Se toma el borde inferior de la vaina anterior del recto con pinzas y se desprende del músculo hasta llegar el pubis exponiendo su porción tendinosa. Los músculos piramidales son seccionados. Se secciona el tendón de los rectos inmediatamente encima del pubis. Los músculos desinsertados se levantan hacia arriba.

El peritoneo ahora expuesto se abre y se confirma la altura de la vejiga, se incide transversalmente $1 \mathrm{~cm}$ sobre la línea de reflexión de ésta con la pared. Se expone el segmento uterino y se realiza una histerotomía convencional.

La síntesis de la pared se realiza por planos. Los músculos piramidales quedan libres. Los ten- 
dones terminales de los rectos pueden o no suturarse. Sugerimos la colocación de puntos en "U" a la porción tendinosa del pubis. La aponeurosis de los músculos oblicuos y la vaina anterior de los rectos se cierra en un plano. El subcutáneo y la piel se suturan de forma convencional.

\section{RESULTADOS}

Durante el período comprendido entre enero de 2004 y junio de 2005 se operaron con técnica de Giordano-Cherney a 7 pacientes con diagnóstico antenatal de malformación del sistema nervioso central. El promedio de edad fue 25,3 años (rango: 21-32 años). Tres pacientes eran primigestas y las restantes multíparas de un parto previo. En todos los casos la intervención se realizó en forma programada con fetos de término, excepto un caso que se interrumpió el embarazo con 31 semanas de gestación. El tiempo promedio de la cirugía completa fue de $72 \pm 11,8$ minutos. El sangrado intraoperatorio no fue cuantificado, sin embargo, ninguna paciente requirió transfusión sanguínea o control médico por la anemia. No se registraron complicaciones intra o post operatorias. El manejo del dolor se realizó en forma rutinaria para una cesárea, con dipirona endovenosa. En 2 pacientes se utilizó ketoprofeno profiláctico en el postoperatorio inmediato. Sólo una paciente requirió control médico postoperatorio y el dolor se manejó satisfactoriamente con opioides. El alta se realizó en promedio tras 3,4 días de post operario. Todas evolucionaron en forma favorable. No se presentaron complicaciones atribuibles a la cirugía en los 7 recién nacidos.

\section{DISCUSIÓN}

La elección de la vía de parto para los fetos con malformación del SNC es controversial. La laparotomía de Giordano-Cherney ofrece considerables ventajas, lo que la ha llevado a ser el abordaje de elección, para estos casos, en nuestro centro.

El diámetro transverso del abdomen es aproximadamente un $25 \%$ mayor a la distancia que se ofrece desde el ombligo a la sínfisis pubiana y mayor aún, que el entregado por la laparotomía de Pfannenstiel. Dado que la exposición del campo es proporcional al cuadrado del eje, esta incisión transversa es capaz de entregar un campo quirúrgico entre 1,5 a 2 veces el que aporta una incisión longitudinal (11). Además, el campo quirúrgico se sitúa en el centro de la incisión y no en el extremo de ésta, lo que entrega una excelente exposición del útero y facilita la extracción fetal, mejorando el resultado obstétrico.

La pared conserva su firmeza considerando que conserva la innervación muscular; las suturas son realizadas en zonas tendinosas, que ofrecen una unión más confiable; las aponeurosis de los oblicuos, rectos y el peritoneo son incididos a distintos niveles, en forma escalonada, evitando un punto de mayor debilidad en la pared. Por último, la dirección de la incisión en la aponeurosis de los músculos oblicuos es en paralelo a la fuerza ejercida en la pared, a lo que se atribuye menor dolor en las incisiones transversas.

La técnica es sencilla, relativamente rápida y se torna fácil de realizar por un operador experimentado. Además, esta incisión resulta atractiva por otras razones: desde un punto de vista cosmético respeta las líneas de Langer (8) y esconde la cicatriz en el límite del vello pubiano; y evita la manipulación visceral reduciendo el íleo post-quirúrgico.

\section{CONCLUSIÓN}

Recomendamos esta técnica quirúrgica porque permite una firme y amplia exposición que no requiere de relajación muscular, es técnicamente simple, relativamente indolora y con buenos resultados cosméticos, asociado a las ventajas obstétricas señaladas.

\section{BIBLIOGRAFÍA}

1. Allan LD. Antenatal diagnosis of congenital heart disease. Heart 2000;83:367-70.

2. Nazer J, Cifuentes L, Rodríguez M, et al. Malformaciones del sistema nervioso central en el Hospita Clínico de la Universidad de Chile y maternidades chilenas participantes en el Estudio Colaborativo Latinoamericano de Malformaciones Congénitas (ECLAMC). Rev Méd Chile 2001;129(10):1163-70.

3. Chervenak FA, Duncan C, Ment LR, Tortora M, McClure M, Hobbins JC. Perinatal management of myelomeningocele. Obstet Gynecol 1984;63:37680.

4. Cochrane D, Aronyk K, Sawatzky B, Wilson D, Steinbok P. The effects of labor and delivery on spinal cord function and ambulation in patients with meningomyelocele. Childs Nerv Syst 1991;7:312-5.

5. Sakala EP, Andree I. Optimal route of delivery for meningomyelocele. Obstet Gynecol Survey 1990;45: $209-21$

6. Luthy DA, Wardinsky T, Shurtleff DB, Hollenbach KA Hickok DE, Nyberg DA, et al. Cesarean section before the onset of labor and subsequent motor 
function in infants with meningomyelocele diagnosed antenatally. N Engl J Med 1991;324:662-6.

7. Chervenak FA, Berkowitz RL, Tortorn $M$, et al. The management of fetal hydrocephalus. Am J Obstet Gynecol 1985;151:933-42.

8. Lee-Parritz A. Surgical techniques for cesarean delivery: what are the best practices? Clin Obstet Gynecol 2004;47(2):286-98.

9. Meeks R, Trenhaile T. Management of abdominal incisions. J Pelvic Surgery 2002;8(6):295-300.
10. Pfannenstiel J. On the advantages of a transverse cut of the fascia above the symphysis for gynecological laparotomies and advice on surgical methods and indications. Samml Klin Votr Gynakol 1897;68-98.

11. Cherney LS. A modified transverse Incision for low abdominal operations. Surg Gynecol Obstet 1941; 72:92-5.

12. Crovetto RM, Stratico JV. Cherney incision in cesarean section. Prensa Med Argent 1957;44(22): 16889. 